\title{
Servo Design for a High Stiffness Linear Repulsive Magnetic-levitation Bearing
}

\author{
Chao-Ming Huang Jia-Yush Yen \\ Graduate Student Professor \\ Department of Mechanical Engineering, \\ National Taiwan University, \\ Taipei, Taiwan 10617, R.O.C. \\ e-mail: Jyen@ccms.ntu.edu.tw
}

\begin{abstract}
This paper presents the servo design of a high stiffness linear repulsive magnetic-levitation (Maglev) bearing. The maglev bearing is a highly nonlinear system with very serious parameter uncertainties. Most existing literatures have discussed the system stability while very few results have talked about the rigidity of the bearing. This paper proposes an adaptive sliding mode controller via input-output feedback linearization (IOFL) process to overcome these difficulties. The system uncertainties are analyzed and a stability proof is presented. A systematic design procedure is then presented. Comparison among the existing experimental results with show that the proposed control has a better performance.
\end{abstract}

\section{Introduction}

As the industrial technology evolves, numerous motion control devices have been designed to meet the demand on servo accuracy. Among them, the suspension systems based on maglev technology played an increasingly important role. If the maglev systems are to be classified by the types of levitation, there can be attractive systems and repulsive systems. Most of the maglev systems discussed in the literature are attractive systems, where attractive forces are applied between the moving carriage and fixed guide rails [1] [5]. The repulsive maglev systems, on the other hand, use repulsive forces to push the moving carriage above the fixed guide rails, and are discussed less often [1], [6], [7], [8].

Maglev technology using two permanent magnets with the same poles facing each other to generate repulsive force is the socalled passive maglev, and using a permanent magnet levitated above a DC electromagnet is the active maglev. The experimental device (Figure 1) used to realize the simulation and real test is a repulsive maglev stage referenced to [7], [8]. Our concept comes from the idea of compounding both active and passive maglev techniques to replace the original design that used the active electromagnetic levitation only. Systems using the active maglev only must apply current to the DC electromagnets at all time to maintain the carriage to fly. This current causes the levitation coils to overheat, liquefies the lacquer protection, and leads to coil short-circuit. The passive maglev can work without these problems; however they are usually lack of performance. Most previous researches in this area have focused on establishing a stable floating condition. In this paper, we take a further step to investigate the critical issues in the precision machine applications. The active components in the system will serve to provide the necessary efforts to achieve the required guide way stiffness and system robustness. The experimental setup in this paper achieves stiffness in the $X$ and $\theta$ direction (Figure 2). The $\phi, \psi$ and $Z$ D-OF's are left free since they are stable by design [7].

It is very hard to design a classical single-input-single-output (SISO) controller for the system due to its highly nonlinear nature. For such class of nonlinear systems, input output feedback linearization (IOFL) technique which has attracted a great deal of attentions in recent years is proposed to be adequate. However, the actual applications resulting in the implementation of such control algorithms have been few due to its computational requirement and limitations. Furthermore, IOFL does not guarantee robustness in the presence of model uncertainties. Recently, the output tracking and stabilization of nonlinear systems with parametric uncertainties have been carried out from a practical perspective based upon adaptive or robust control scheme $[11,13,14]$. Unfortunately, these control laws are very complicated and most available demonstrations are limited to simple systems. Because a precise mathematical model is not always available, these methods suffer lose of the physical senses between the adaptation mechanism and the system responses.

What we need in cases of more complex systems is to find the sources of uncertainties and use an easily implemented controller. Our solution is to combine the sliding mode control (SMC) and the IOFL with a slight touch of adaptive purpose. Where the IOFL could de-couple the system dynamics, the SMC serves as a powerful robust controller, and the adaptive feature could help us obtain the best performance. In this paper, a precise model for the maglev suspension guide rail will be derived. We will analyze the sources of the system uncertainties. The controller synthesis of an adaptive sliding mode controller via IOFL for an MIMO second order nonlinear system will be presented. Experimental exercises will then demonstrate the effectiveness of the control.

\section{Experimental Device and Model}

A knowledge model of the maglev suspension system is obtained using Newton-Euler equations of motion for a body that moves in space (Figure 3), and a set of differential equations is found with the following form

$$
\begin{aligned}
& \ddot{\theta}=\frac{1}{J_{z}}\left\{\left(-a_{1}+b_{1} \theta\right)\left(F_{A S X}+F_{B S X}+F_{\text {MAX }}+F_{B A X}\right)\right. \\
& \left.+\left(a_{2}+b_{2} \theta\right)\left(F_{C S T}+F_{\text {DST }}+F_{C M Y}+F_{\text {DAX }}\right)\right\}
\end{aligned}
$$

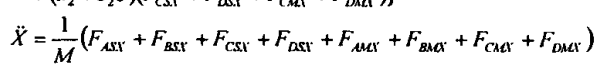

where

$$
\begin{aligned}
& F_{A S x}=N_{s} \frac{\mu_{0} I_{\text {sabt }}}{2 \pi}\left\{\frac{-\left(d+\delta A_{y}\right)^{2} \Omega_{z}}{\left[\left(d+\delta A_{y}\right)^{2}\right]^{2}}+\frac{-\left(-d+\delta A_{x}\right)^{2} \Omega_{z}}{\left[\left(-d+\delta A_{x}\right)^{2}\right]^{2}}\right\}=K_{\text {ASS }} I_{\text {stabl }} \\
& F_{\text {BSX }}=N_{s} \frac{\mu_{0} I_{\text {tatabl }}}{2 \pi}\left\{\frac{-\left(d+\delta B_{X}\right)^{2} \Omega_{Z}}{\left[\left(d+\delta B_{X}\right)^{2}+\delta B_{Z}^{2}\right]^{2}}+\frac{-\left(-d+\delta B_{X}\right)^{2} \Omega_{Z}}{\left[\left(-d+\delta B_{X}\right)^{2}\right]^{2}}\right\}=K_{B S X} I_{\text {.tabl }} \\
& F_{C S S}=N_{s} \frac{\mu_{0} I_{\text {sati } 2}}{2 \pi}\left\{\frac{-\left(d+\delta C_{X}\right)^{2} \Omega_{Z}}{\left[\left(d+\delta C_{X}\right)^{2}\right]^{2}}+\frac{-\left(-d+\delta C_{X}\right)^{2} \Omega_{Z}}{\left[\left(-d+\delta C_{X}\right)^{2}\right]^{2}}\right\}=K_{c s S} I_{\text {stah } 2} \\
& F_{\text {osi }}=N_{s} \frac{\mu_{0} I_{\text {sob2 }}}{2 \pi}\left\{\frac{-\left(d+\delta D_{x}\right)^{2} \Omega_{z}}{\left[\left(d+\delta D_{x}\right)^{2}\right]^{2}}+\frac{-\left(-d+\delta D_{x}\right)^{2} \Omega_{z}}{\left[\left(-d+\delta D_{X}\right)^{2}\right]^{2}}\right\}=K_{\text {osr }} I_{\text {subs }}
\end{aligned}
$$

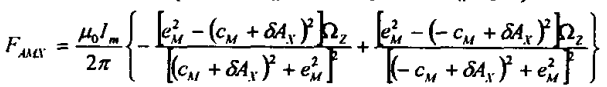

$$
\begin{aligned}
& F_{B A X}=\frac{\mu_{0} I_{m}}{2 \pi}\left\{-\frac{\left[e_{M}^{2}-\left(c_{M}+\delta B_{X}\right)^{2} \mathbf{k}_{\mathrm{Z}}\right.}{\left[\left(c_{M}+\delta B_{X}\right)^{2}+e_{M}^{2}\right]^{2}}+\frac{\left[e_{M}^{2}-\left(-c_{M}+\delta B_{X}\right)^{2} \mathbf{k}_{z}\right.}{\left[\left(-c_{M}+\delta B_{X}\right)^{2}+e_{M}^{2}\right]^{2}}\right\}
\end{aligned}
$$




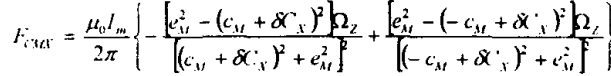

$$
\begin{aligned}
& F_{\text {DuU }}=\frac{\mu_{0} I_{m}}{2 \pi}\left\{-\frac{\left[c_{M}^{2}-\left(c_{M}+\delta D_{x}\right)^{2} \mathbf{2}_{z}\right.}{\left[\left(c_{M}+\delta D_{x}\right)^{2}+e_{M}^{2}\right]^{2}}+\frac{\left(k_{M}^{2}-\left(-c_{M}+\delta D_{x}\right)^{2} \mathbf{2}_{z}\right.}{\left[\left(-c_{M}+\delta D_{x}\right)^{2}+e_{M}^{2}\right]^{2}}\right\} \\
& \delta A_{x}=-a_{1} \theta+X \\
& \delta B_{x}=-a_{1} \theta+\chi \\
& \delta C_{X}=u_{2} \theta+X \\
& \delta D_{x}=a_{2} \theta+X
\end{aligned}
$$

All model parameters are listed in Table 1 . The readers can refer to [7]. [8] for some of the details. The model presented here also includes the passive levitator. The magnetic flux density from the passive levitator is modeled by the magnetic field generated by a coil around the track that carries an equivalent current, the socalled Magnetization Current $I_{m}$, see [9]. Apply $I_{m}$ into the force and torque models in [7] and [10] to get the nonlinear forces $F_{A M X}$, $F_{B M X}, F_{C M X}$ and $F_{D M X}$. The results are given with the applied currents $I_{\text {stabl }}$ and $I_{\text {stabl }}$, which are also used as the system inputs. The general form of the system can be rearranged to give

$$
\begin{aligned}
& \ddot{\zeta}_{1}=f_{1}\left(\zeta_{1}, \zeta_{2}\right)+g_{11}\left(\zeta_{1}, \zeta_{2}\right) I_{\text {satab }}+g_{12}\left(\zeta_{1}, \zeta_{2}\right) I_{\text {sab2 }} \\
& \ddot{\zeta}_{2}=f_{2}\left(\zeta_{1}, \xi_{2}\right)+g_{21}\left(\zeta_{1}, \zeta_{2}\right) I_{\text {stabl }}+g_{22}\left(\zeta_{1}, \zeta_{2}\right) I_{\text {stab2 } 2}
\end{aligned}
$$

where $\zeta_{1}=\theta, \zeta_{2}=X . f_{1}, f_{2}, g_{11}, g_{12}, g_{21}, g_{2} 2$ are functions of $\zeta_{1}, \zeta_{2}$.

\section{Uncertainty Analysis}

The mathematical model for the maglev suspension system is presented in the previous section. Parameters like $a_{1}, a_{2}, b_{1}, b_{2}, d$, $c_{M}$, and $d_{M}$ are the dimensions of the system. The variation of these parameters plays less important roles affecting the system stability and rigidity performance. They are also more controllable with the precision manufacturing process. $N_{S}$ is the number of turns of the stabilizer coils and is precise. The mass and the inertia of the carriage, $M$ and $J_{z}$, and the magnetization properties of the rail, $\Omega_{z}$ and $I_{m}$, play important roles in affecting the system performance and are the sources of the system uncertainties.

By substituting $\Omega_{Z}, I_{m}, M$ and $J_{Z}$, equation (2) can be rewritten as

$$
\begin{aligned}
& \ddot{\zeta}_{1}=\frac{I_{m} \Omega_{z}}{J_{z}} f_{\text {mominal.1. }}(\zeta)+\frac{\Omega_{z}}{J_{z}} g_{\text {momina }, 111}(\zeta) I_{\text {stabl }}+\frac{\Omega_{z}}{J_{z}} g_{\text {momina }, 12}(\zeta) I_{\text {sabz }}
\end{aligned}
$$

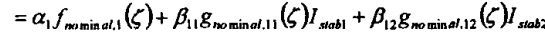

$$
\begin{aligned}
& \ddot{\zeta}_{2}=\frac{I_{m} \Omega_{z}}{M} f_{\text {moninal.2. }}(\zeta)+\frac{\Omega_{z}}{M} g_{\text {momina } t, 21}(\zeta) I_{\text {stab1 }}+\frac{\Omega_{z}}{M} g_{\text {mominad }, 22}(\zeta) I_{\text {stab } 2} \\
& =\alpha_{2} f_{\text {mominal. } 22}(\zeta)+\beta_{21} g_{\text {mominat.21 } 21}(\zeta) I_{\text {stabl }}+\beta_{22} g_{\text {mominat }, 22}(\zeta) I_{\text {stab2 }}
\end{aligned}
$$

where $\alpha_{1}, \alpha_{2}, \beta_{11}, \beta_{12}, \beta_{21}$ and $\beta_{22}$ are treated as the unknown parameters. Ideally, $\beta_{11}$ and $\beta_{21}$ should be equal to $\beta_{12}$ and $\beta_{22}$, but it is almost impossible to get identical magnets for the four tracks. Therefore, it is better to regard $\beta_{11}, \beta_{12}, \beta_{21}$ and $\beta_{22}$ as individual variables.

The following section will focus on the subject of applying adaptive sliding mode control via IOFL to the dynamical system with unknown parameters.

\section{Adaptive Sliding Mode Control of Nonlinear System with Unknown Parameters}

In this section we describe a systematic algorithm for the adaptive sliding mode output tracking control. Our control law is a modification of the dynamical adaptive sliding mode output tracking control in [11]. The algorithm is suitable for second order MIMO nonlinear systems, which are input-output linearizable, and with constant, but unknown parameters. This class of systems can be represented by the following system equations

$$
\begin{aligned}
& \left\{\begin{array}{l}
\ddot{\zeta}_{1}=\alpha_{1} f_{1}(\zeta)+\beta_{11} g_{11}(\zeta) u_{1}+\ldots+\beta_{1 n} g_{1 n}(\zeta) u_{n} \\
\vdots \\
\ddot{\zeta}_{n}=\alpha_{n} f_{n}(\zeta)+\beta_{m 1} g_{n 1}(\zeta) u_{1}+\ldots+\beta_{m n} g_{m}(\zeta) u_{n}
\end{array}\right. \\
& \left\{\begin{array}{c}
h_{1}(\zeta) \\
\vdots \\
h_{u}(\zeta)
\end{array}\right]=\left[\begin{array}{c}
\zeta_{1} \\
\vdots \\
\zeta_{n}
\end{array}\right]
\end{aligned}
$$

Equation (4) is frequently used in most mechanical positioning system like X-Y table, rigid robot manipulators, and our maglev suspension system. In (4),

$$
\zeta=\left[\begin{array}{lllll}
\zeta_{1} & \zeta_{1} & \cdots & \zeta_{n} & \dot{\zeta}_{n}
\end{array}\right]^{\top} \in \mathfrak{P}^{2 n}
$$

are states of system,

$$
u=\left[\begin{array}{lll}
u_{1} & \ldots & u_{n}
\end{array}\right]^{\top} \in \mathfrak{R}^{n}
$$

are control inputs of system, and

$$
y=\left[\begin{array}{lll}
h_{1}(\zeta) & \ldots & h_{n}(\zeta)
\end{array}\right]^{\top} \in \mathfrak{R}^{n}
$$

are the system measurement outputs. $\alpha_{1} \sim \alpha_{n}$ and $\beta_{11} \sim \beta_{r n}$ are the unknown parameters. We assume that $f_{1} \sim f_{n}, g_{11} \sim g_{n n}$ are smooth field and the relative degree of equation (4) are well-defined. The control objective is to drive the system output $y(t)$ to track asymptotically a desired reference signal $y_{r}(t)$. We also assume that $y_{r}(t)$ and its derivatives up to order two are bounded and are sufficiently smooth functions of $t$.

\section{Step 1. Design of Parameters Estimate System}

According to the nominal system in equation (4), we design an estimated system

$$
\begin{aligned}
& \left\{\begin{array}{l}
\ddot{\zeta}_{1}=\bar{\alpha}_{1} f_{1}(\zeta)+\hat{\beta}_{11} g_{11}(\zeta) u_{1}+\ldots+\hat{\beta}_{1 n} g_{1 n}(\zeta) u_{n} \\
\vdots \\
\ddot{\zeta}_{n}=\bar{\alpha}_{n} f_{n}(\zeta)+\bar{\beta}_{n 1} g_{n 1}(\zeta) u_{1}+\ldots+\hat{\beta}_{n n} g_{n n}(\zeta) u_{n}
\end{array}\right. \\
& \left\{\begin{array}{c}
h_{1}(\zeta) \\
\vdots \\
h_{n}(\zeta)
\end{array}\right]=\left[\begin{array}{c}
\zeta \\
\vdots \\
\zeta_{n}
\end{array}\right]
\end{aligned}
$$

where $\hat{\alpha}_{1}, \ldots, \bar{\alpha}_{n}, \hat{\beta}_{11}, \cdots, \hat{\beta}_{n n}$ are estimates of $\alpha_{1} \sim \alpha_{n}$ and $\beta_{11} \sim \beta_{n n}$

Step 2. Input-Output Feedback Linearization of Real System and Estimate System

From the input-output feedback linearization technique, we can obtain a coordinate transformation to de-couple both Equation (4) and (5) by introducing new control input $v$.

$$
v=\left[\begin{array}{c}
v_{1} \\
\vdots \\
v_{n}
\end{array}\right]=\left[\begin{array}{c}
\hat{\alpha}_{1} f_{1}(\zeta) \\
\vdots \\
\hat{\alpha}_{n} f_{n}(\zeta)
\end{array}\right]+\left[\begin{array}{ccc}
\hat{\beta}_{11} g_{11}(\zeta) & \ldots & \hat{\beta}_{1 n} g_{1 n}(\zeta) \\
\vdots & \ddots & \vdots \\
\hat{\beta}_{n 1} g_{m 1}(\zeta) & \cdots & \hat{\beta}_{n n} g_{n n}(\zeta)
\end{array}\right]\left[\begin{array}{c}
u_{1} \\
\vdots \\
u_{n}
\end{array}\right]
$$

The de-coupled estimate system will have the following form

$$
\left[\begin{array}{c}
\ddot{\zeta}_{1} \\
\vdots \\
\ddot{\zeta}_{n}
\end{array}\right]=\left[\begin{array}{c}
v_{1} \\
\vdots \\
v_{n}
\end{array}\right]
$$

and the de-coupled nominal system can be rewritten as

$$
\begin{aligned}
{\left[\begin{array}{c}
\ddot{\zeta}_{1} \\
\vdots \\
\ddot{\zeta}_{n}
\end{array}\right] } & =\left[\begin{array}{c}
v_{1} \\
\vdots \\
v_{n}
\end{array}\right]-\left[\begin{array}{c}
\tilde{\alpha}_{1} f_{1} \\
\vdots \\
\tilde{\alpha}_{n} f_{n}
\end{array}\right] \\
& +\left[\begin{array}{ccc}
\tilde{\beta}_{11} g_{11} & \cdots & \tilde{\beta}_{1 n} g_{1 n} \\
\vdots & \ddots & \vdots \\
\tilde{\beta}_{n 1} g_{n 1} & \cdots & \tilde{\beta}_{n n} g_{n n}
\end{array}\right]\left[\begin{array}{ccc}
\hat{\beta}_{11} g_{11} & \cdots & \hat{\beta}_{1 n} g_{1 n} \\
\vdots & \ddots & \vdots \\
\hat{\beta}_{n 1} g_{n 1} & \cdots & \hat{\beta}_{n n} g_{n n}
\end{array}\right]^{-1}\left[\begin{array}{c}
\tilde{\alpha}_{1} f_{1}-v_{1} \\
\vdots \\
\bar{\alpha}_{n} f_{n}-v_{n}
\end{array}\right]
\end{aligned}
$$

where $\widetilde{\alpha}_{i}=\hat{\alpha}_{i}-\alpha_{i}, i=1, \ldots, n, \widetilde{\beta}_{j, k}=\hat{\beta}_{j, k}-\beta_{j, k}, j=1, \ldots, n, k=1, \ldots, n$. We can rewrite Equation (8) to the following form

$$
\left[\begin{array}{c}
\ddot{\zeta}_{1} \\
\vdots \\
\ddot{\zeta_{n}}
\end{array}\right]=\left[\begin{array}{c}
v_{1} \\
\vdots \\
v_{n}
\end{array}\right]+\left[\begin{array}{c}
\Phi_{1}^{T} \omega_{1} \\
\vdots \\
\Phi_{n}^{T} \omega_{n}
\end{array}\right]
$$




$$
\begin{aligned}
& \Phi_{1}=\left[\begin{array}{llll}
\tilde{\alpha}_{1} & \tilde{\beta}_{i 1} & \cdots & \widetilde{\beta}_{i n}
\end{array}\right]^{T} \\
& \omega_{i}=\left[\begin{array}{llll}
f_{i} & g_{i 1} \Delta_{1} & \cdots & g_{i n} \Delta_{n}
\end{array}\right]^{T} \\
& {\left[\begin{array}{c}
\Delta_{1} \\
\vdots \\
\Delta_{n}
\end{array}\right]=\left[\begin{array}{ccc}
\bar{\beta}_{11} g_{11} & \cdots & \bar{\beta}_{i n} g_{1 n} \\
\vdots & \ddots & \vdots \\
\tilde{\beta}_{m 1} g_{n 1} & \cdots & \hat{\beta}_{m u} g_{m n}
\end{array}\right]^{-1}\left[\begin{array}{c}
\bar{\alpha}_{1} f_{1}-v_{1} \\
\vdots \\
\hat{\alpha}_{n} f_{n}-v_{n}
\end{array}\right]}
\end{aligned}
$$

Step 3. Adaptive Sliding Output Tracking Controller Design

Define the error co-ordinates by

$$
\begin{aligned}
& z_{i, 1}=h_{i}(\zeta)-y_{i, r}(t)=\zeta_{i}-y_{t, r}(t) \\
& z_{i .2}=\left[\dot{\zeta}_{i}-\dot{y}_{i, r}(t)\right]+\zeta_{i}-y_{i, r}(t) \\
& i=1, \ldots, n
\end{aligned}
$$

where $y_{\mathrm{ir}}(t)$ are the reference commands. The asymptotic outputtracking objective is achieved by specifying the sliding surfaces in terms of the error co-ordinates, $z_{i, 1}$ and $z_{i, 2}$.

$$
S_{i}=z_{i, 2}+\lambda_{i}=z_{i, 1},
$$

$$
i=1, \ldots, n
$$

where the scalar coefficient $\lambda_{i}$ must have positive real part. Using the update law

$$
\dot{\Phi}_{i}=-S_{1} \Gamma_{i} \omega_{i}
$$$$
i=1, \ldots, n
$$

and together with the adaptive sliding mode output tracking controller

$$
v_{i}=-\left(1+\lambda_{i}\right)_{i, 2}+\left(1+\lambda_{i}\right) z_{i, 1}-K_{i}\left[S_{i}+W_{i} \operatorname{sign}\left(S_{i}\right)\right]
$$

with $K_{i}>0, W>0, \Gamma_{i}=\Gamma_{i}^{\top}>0$, a matrix of adaptation gains.

Step 4. Apply Control Inputs to System

The control inputs of Equation (9) are obtained and then transformed to real inputs by Equation (15).

$$
\left[\begin{array}{c}
u_{1} \\
\vdots \\
u_{n}
\end{array}\right]=\left[\begin{array}{ccc}
\hat{\beta}_{11} g_{11}(\zeta) & \ldots & \bar{\beta}_{1 n} g_{1 n}(\zeta) \\
\vdots & \ddots & \vdots \\
\hat{\beta}_{n 1} g_{n 1}(\zeta) & \cdots & \hat{\beta}_{n m} g_{m i}(\zeta)
\end{array}\right]^{-1}\left(\left[\begin{array}{c}
v_{1} \\
\vdots \\
v_{n}
\end{array}\right]-\left[\begin{array}{c}
\bar{\alpha}_{1} f_{1}(\zeta) \\
\vdots \\
\hat{\alpha}_{n} f_{n}(\zeta)
\end{array}\right]\right)
$$

The details of the systematic approach are listed in the Appendix. The result can be applied to Equation (3) directly.

\section{Stability Analysis}

Assume the relative degree of the system is well-defined. This control law is suitable for many positioning control problems.

Step 1 .

Define the output tracking errors as in (11), then we have the directives of $z_{i, 1}, z_{i, 2}$.

$$
\begin{aligned}
& \dot{z}_{i .1}=z_{i .2}-z_{i .1} \\
& \dot{z}_{i .2}=\ddot{\zeta}_{i}-\ddot{y}_{i, r}(t)+\dot{z}_{i .1}=v_{i}+\Phi_{i}^{T} \omega_{i}-\ddot{y}_{i . r}(t)+z_{i .2}-z_{i .1} \\
& i=1, \ldots, n
\end{aligned}
$$

Step 2.

Consider the quadratic Lyapunov function:

$$
\begin{aligned}
& L_{i}=\frac{1}{2} z_{i, 1}^{2}+\frac{1}{2} \Phi_{i}^{T} \Gamma_{i}^{-1} \Phi_{i}+\frac{1}{2} S_{i}^{2} \geq 0 \\
& i=1, \ldots, n
\end{aligned}
$$

where $\Gamma=\Gamma^{\top}>0$, and $S_{i}$ is defined in Equation (12). The time directive of $L_{i}$ is :

$$
\begin{aligned}
\dot{L}_{i} & =z_{i, 1} \dot{z}_{i, 1}+\Phi_{i}^{T} \Gamma_{i}^{-1} \dot{\Phi}_{i}+S_{i} \dot{S}_{i} \\
& =-z_{i, 1}^{2}+z_{i, 1} z_{i, 2}+\Phi_{i}^{T} \Gamma_{i}^{-1} \dot{\Phi}_{i}+S_{i}\left\{\Phi_{i}^{T} \omega_{i}-K_{i}\left[S_{i}+W_{i} \operatorname{sign}\left(S_{i}\right)\right]\right\} \\
& =-z_{i .1}^{2}+z_{i, 1} z_{i .2}+\Phi_{i}^{T} \Gamma_{i}^{-1}\left(\Phi_{i}+S_{i} \Gamma_{i} \omega_{i}\right)-K_{i} S_{i}^{2}-K_{i} W_{i}\left|S_{i}\right|
\end{aligned}
$$

With updating law (13), we will have:

$$
\begin{aligned}
& \dot{L}_{i}=-z_{i, 1}^{2}+z_{i .1} z_{i .2}-K_{i} S_{i}^{2}-K_{i} W_{i}\left|S_{i}\right| \\
& =-z_{i, 1}^{2}+z_{i .1} z_{i, 2}-K_{i}\left(z_{i, 2}+\lambda_{i} z_{i, 1}\right)^{2}-K_{i} W_{i}\left|S_{1}\right|
\end{aligned}
$$

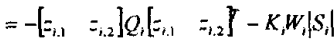

where

$$
Q_{i}=\left[\begin{array}{cc}
1+K_{i} \lambda_{i}^{2} & -\frac{1}{2}+K_{i} \lambda_{i} \\
-\frac{1}{2}+K_{i} \lambda_{i} & K_{i}
\end{array}\right]
$$

where the eigenvalues of $Q_{i}$ are

$$
\frac{1}{8}\left(4+4 K_{i}+4 K_{i} \lambda_{i}^{2} \pm \sqrt{-16\left(-1+4 K_{i}+4 K_{i} \lambda_{i}\right)+\left(-4-4 K_{i}-4 K_{i} \lambda_{i}^{2}\right)^{2}}\right)
$$

Thus, a sufficient condition on the design parameters to achieve asymptotic tracking can be obtained from:

$$
-1+4 K_{i}+4 K_{i} \lambda_{i}>0
$$

So $\dot{L}_{i} \leq 0$ and, therefore, since

$$
\lim _{t \rightarrow \infty} z_{t, 1}(t)=y_{1}(t)-y_{t, r}(t)=0
$$

Asymptotic tracking is achieved.

The advantages of this control algorithm are that the output tracking error function $z_{i, 1}$ asymptotically approaches zero with substantially reduced chattering.

\section{Experimental Results and Discussion}

\section{Experimental Configuration}

In this section, the maglev suspension system is studied experimentally to compare the performance of the adaptive sliding mode controller via IOFL with the results in [7], [8]. The maglev suspension system consists of the mechanism in Figure 1, a set of the coil power amplifiers, a Pentium $233 \mathrm{PC}$ for controller implementation, a set of inductive gauging sensors for attitude measurement, 12-bit AD converter and a 12-bit DA converter as system $1 / O$ devices. The sensor resolution is $2 \mu \mathrm{m}$ and the bandwidth of the sensors is $3.3 \mathrm{kHz}$. The control inputs are limited to within $\pm 2 A m p$ to protect the levitation coils. Due to the computational power of Pentium 233 PC, high-speed sampling is possible when complex control algorithm is implemented and the sampling error can be neglected. The experimental setup is shown in Figure 5.

\section{Measurement System}

The states $\theta$ and $X$ can not be measured from the sensors directly. An alternate strategy often adopted in the maglev system is to measure gaps between the carriage and some reference planes by means of displacement sensors. According to the design preliminaries in [12] and the fact that the size of the carriage is smaller than the range of the motion, the sensor is mounted on the carriages and the straightedge is fixed to a reference coordinate system (bottom). As a result, it is relatively easy to mount the straightedge, but then one must contend with the flexing of the sensor cable, which causes undesirable effect to the system dynamics. Figure 2 shows the locations of the sensors and the reference plane; the states $\theta$ and $X$ can be obtained from the gap distances. Because each sensor shows inconsistent nonlinearity and DC offset according to the environment, these sensors have to be experimentally calibrated as functions of the $A D$ converters' output value before the experiment is started.

Experimental Results

The goals set for the maglev suspension system are the demonstration of steady-state position stability, de-coupling of the degrees of freedom, improvement of rail rigidity, and the robustness to system uncertainties. According to the control algorithm derived in previous section, the preceding demands and the elimination of chatting will be expected.

To make sure the control framework can introduce the maglev suspension system into stable regulation from its nonlinear nature, the responses with respect to the four initial conditions (Figure 4) should be taken into consideration. The reasons are that the dynamics in the $\theta$ and $X$ direction are coupled with each other and have different response speeds with respect to the control inputs. A controller failed to de-couple the dynamics may not guarantee the convergence of both states within the same settling time. The experiment results will show that the adaptive sliding mode control via IOFL could settle both dynamics in within $0.1 \mathrm{sec}$ with 
respect to the $0.75 \mathrm{sec}$ in [8] and $0.6 \mathrm{sec}$ in [7].

The experimental results should be divided into four parts for testing of each case. however, because of space limitation, only the results of cases $A$ and $C$ are shown. Figure 6 is the result of the response with the maximum positive displacement in the initial conditions Case A. Figure 7 is the situation with maximum positive rotation in Case $C$. The parameters of the controller are $\lambda_{1}=200, \lambda_{2}=200$ (fifth of the sampling frequency), $W_{1}=30, W_{2}=4$, $K_{1}=K_{2}=0.01, \Gamma_{1}$ and $\Gamma_{1}$ are both $3 \times 3$ identity matrices. And the initial conditions of the uncertainty estimates are the values based on the original design and can't set to zeros

\section{Conclusions}

In this paper, we characterized the class of feedback linearizable uncertain nonlinear plants and showed that a plant in this class can be linearized and stabilized using the adaptive sliding mode control via IOFL. We also showed that the maglev suspension system belong to this class. Thus, the adaptive sliding mode control via IOFL is proper. The paper moves further to analyze the robustness property of the controlled system. The implementation of the proposed control law on a PC based system is also presented. Some tests were conducted for the experimental comparison with other results in [7] and [8]. The experimental results demonstrate that the proposed control shows better performance than the control laws in [7], [8].

\section{Acknowledgements}

This work is supported by the National Science Council, Taiwan R.O.C. under project No. NSC87-2213-E-002-091.

\section{Reference}

[1] P. K. Sinha, Electromagnetic Suspension, "Dynamics and Control". London. Peter Peregrinus, 1987.

[2] D. Cho, Y. Kato, and D. Spilman, "Sliding Mode and Classical Control of Magnetic Levitation Systems". IEEE Control Syst. Mag., vol. 13,no. 1, pp. 42-48, 1993.

[3] D. L. Atherton, "Maglev using paermagnets", IEEE Trans. Magn., vol. 16,no. 1., pp. 146-148, 1980.

[4] M. Morishita, T. Azukizawa, S. Kanda, N. Tamura, and T. Yokoyama, "A new maglev system for magnetically levitated carrier system", 1986 Int. Conf. Maglev and Linear Drives, Vancouver, B. C., May 1986.

[5] O. Tsukamoto, K. Yasuda, and J. Z. Chen, "A New Magnetic Levitation System with ac Magnets", IEEE Trans. Magn., vol. 24, no. 2, pp. 1497-1500, 1988.

[6] R. Williams, J. R. Matey, Y. Arie, and J. Rathee, "The effect of mass and pole strength on the levitation height of the magnet over a superconductor", J. App. Phys., vol. 65, no. 9, pp. 3583-3585, 1989.

[7] I.Y. Wang. A Magnetic Levitation Silicon Wafer Transport System. Ph.D. Thesis, The University of Texas at Austin, 1993.

[8] K. N. Wu, and L. L. Chen, "Adaptive control of a four-track maglev system", Journal of Control Systems and Technology 4, 4,pp. 295-302, 1996.

[9] E. M. Purcell, Electricity and Magnetism, Berkeley Physics Course vol 2. McGraw-Hill, 1965.

[10] J. G. David, Introduction to Electrodynamics, Prentice-Hall, Inc., Englewood Cliffs, New Jersey, 1981

[11] M. Rios-Bolivar, A. S. I. Zinober, and H. Sira-Ramirez, "Dynamical adaptive sliding mode output tracking control of a class of nonlinear systems", Int. J. Robust Nonlinear Control, Vol. 7, pp. 387-405, 1997.

[12] Alexander H. Slocum, "Precision Machine Design", Prentice Hall, 1992.

[13] Le Yi Wang, Imad Makki and Wei Zhan, "A Note on Robust Stabilization of Feedback Linearization Systems", Int. J. Robust Nonlinear Control, Vol. 7, pp. 85-95, 1997.

[14] Riccard Marino and Patrizio Tomei, "Robust Adaptive State-Feedback Tracking for Nonlinear Systems", IEEE Trans. Automatic Control, Vol. 43, No. 1, pp. 84-89, 1998.

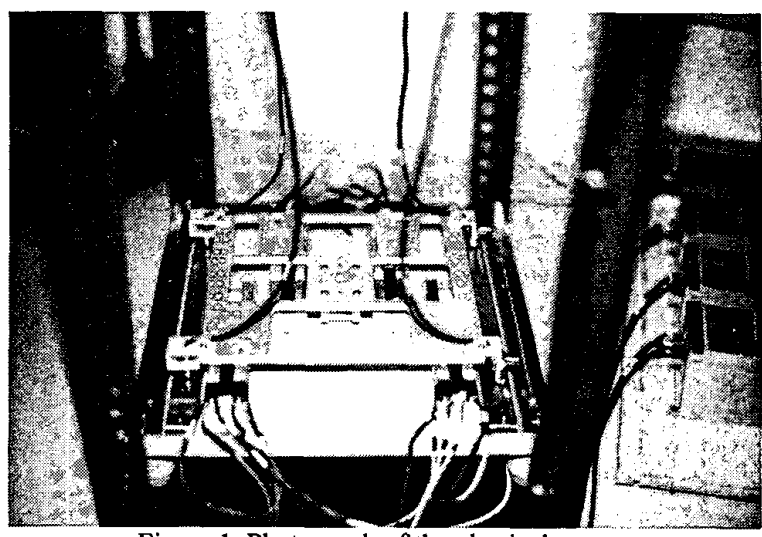

Figure 1. Photograph of the physical system

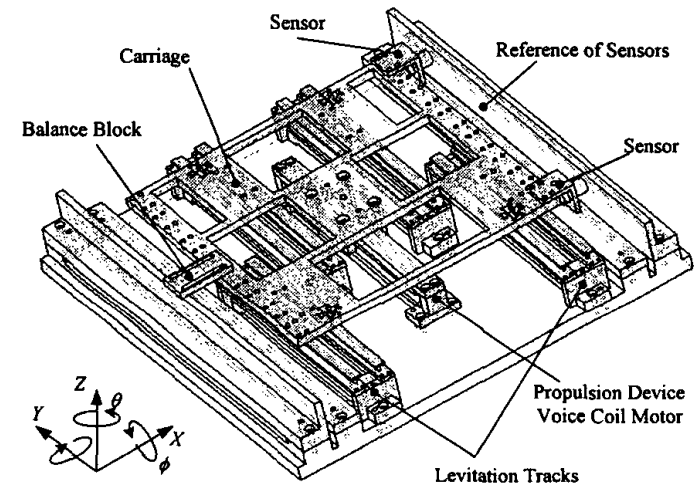

Figure 2. A 3-D view of a section of the entire system.

Table 1: Parameters list of the model

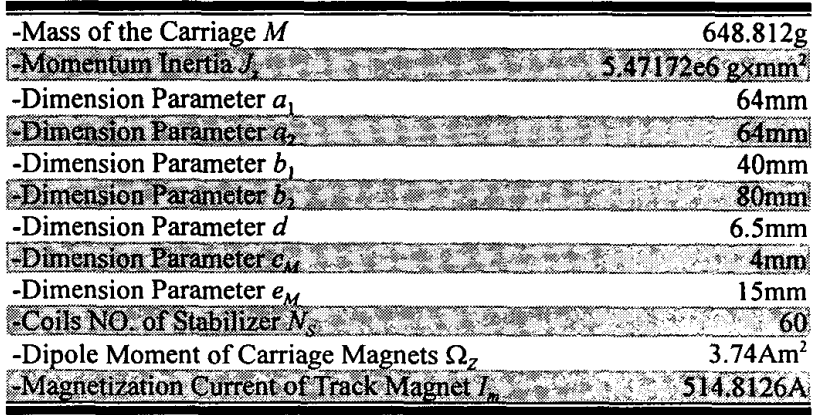




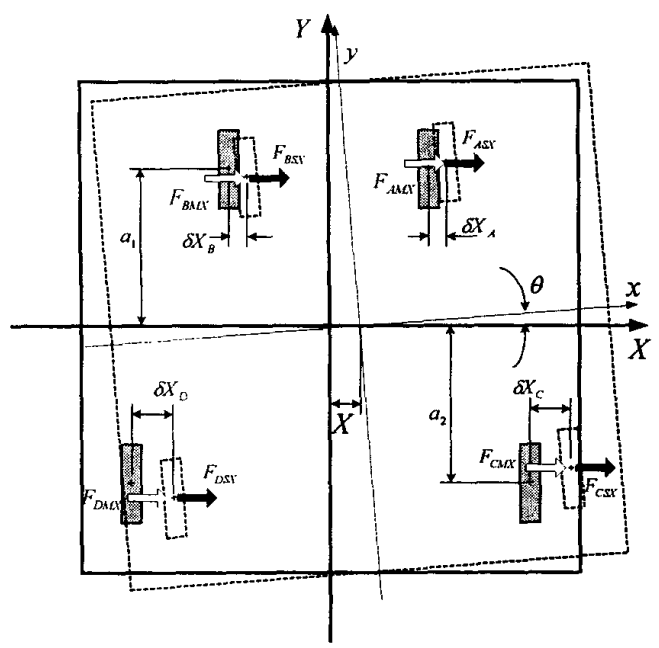

Figure 3. The free body diagram of the carriage after undergoing $X$ translation and $\theta$ rotation.

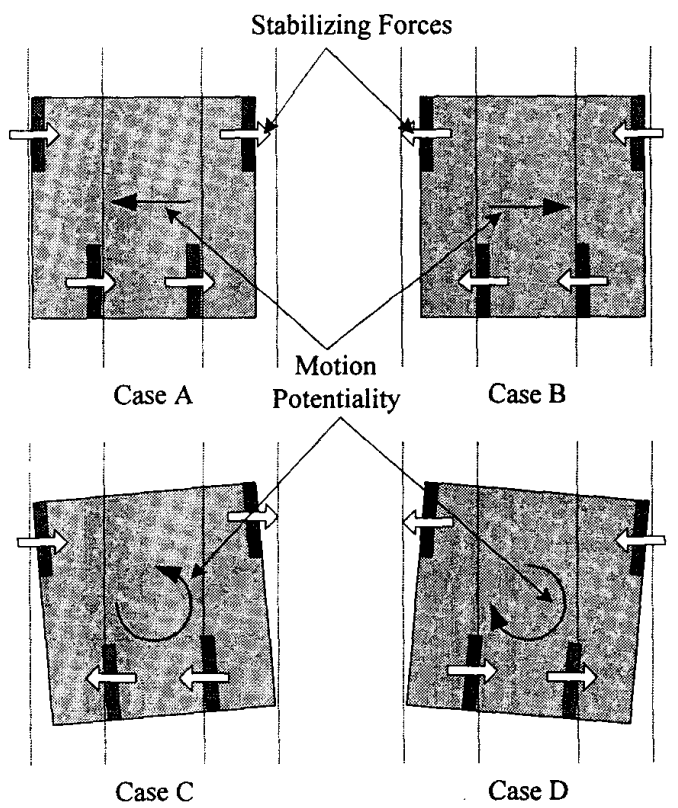

Figure 4. Four typical cases of initial condition (IC). Where Case $A$ is the maximum positive translation IC, Case $B$ is the maximum negative translation $\mathrm{IC}$, Case $C$ is the maximum positive rotation IC, and Case $\mathrm{D}$ is the maximum negative rotation IC.

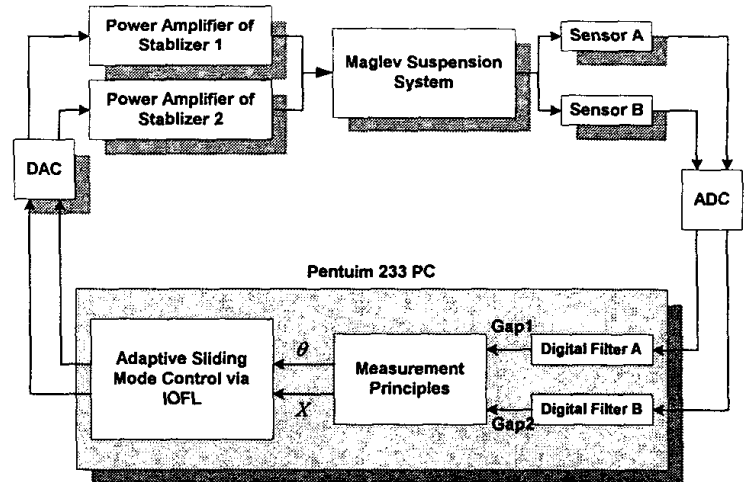

Figure 5. Block diagram of the closed loop system.
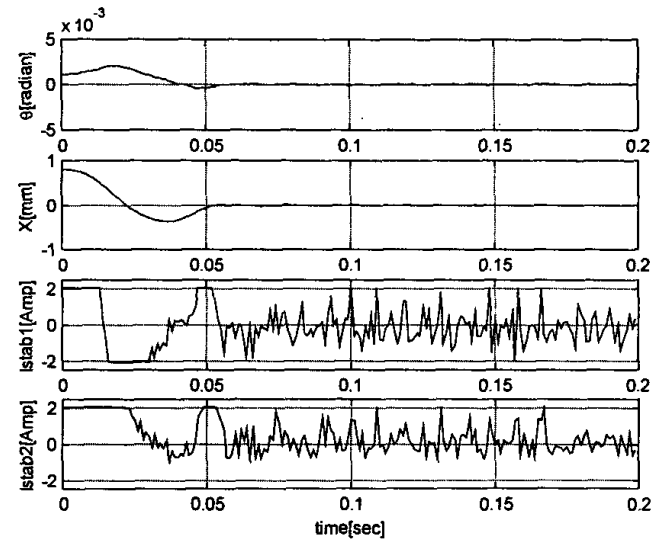

Figure 6. The experimental result of IC Case A, maximum positive translation displacement.

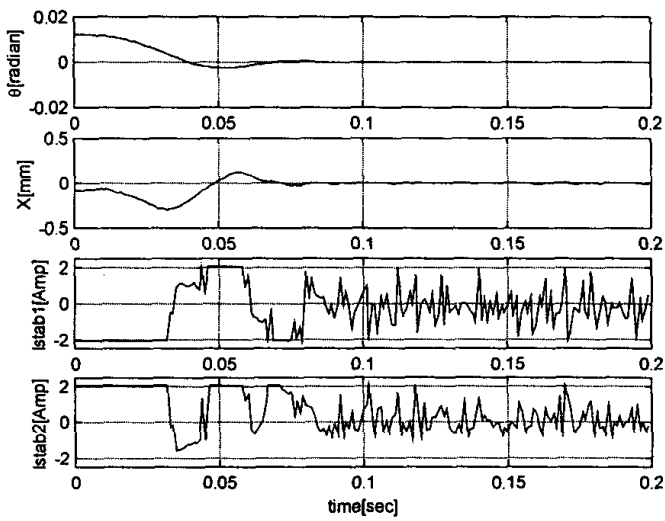

Figure 7. The experimental result of IC Case $C$, maximum positive rotation displacement. 